\title{
Simulation and Optimization of MCM Interconnections*
}

\author{
Wu-shiung Feng, Shing Tenqchen*, and Ming-chi Chen \\ Department of Electrical Engineering \\ National Taiwan University, Taipei, Taiwan, ROC ${ }^{*}$ \\ *CHTTL-VLSI Center, 12, Lane 551, Min-Tsu Rd. Sec. 3, \\ Yang-Mei Zien, Tao-Yuan County, Taiwan, ROC \\ Tel: 886-2-23635251-244 \\ FAX: 886-2-23638247 \\ E-mail: fengws@cc.ee.ntu.edu.tw
}

\begin{abstract}
In this work, we develop a dedicated simulation system to analyze MCM interconnection networks and help the package designers to find the optimal design. This simulation system consists of three parts, namely, parameter calculator, circuit simulator, and circuit optimizer. The parameter calculator evaluates the transmission-line parameters of interconnections. These parameters are fed into the circuit simulator to determine the time-domain response of an MCM interconnection network. If the circuit response does not satisfy the performance specifications, the circuit optimizer can help us to find the optimal geometric parameters of interconnections.
\end{abstract}

Key words: Simulation, Optimization, MCM, Interconnection

\section{Introduction}

Advances in the speed and density of integrated circuits used in high-performance systems have created a greater demand for higher density packaging to improve electrical performance. To meet this demand, multichip module (MCM) technologies [1] have been developed. In the MCM, bare chips are interconnected on a substrate with substantially finer conductor lines, smaller dielectric thickness, and denser via grid than those of boards, and thus reduce the interconnection delay between chips. However, to maintain signal fidelity at high clock rates, interconnections must be designed as impedancecontrolled transmission lines with proper concern for discontinuity, crosstalk, and attenuation [2-3]. Thus, CAD tools are becoming increasingly important for simulating the electrical performance of interconnections prior to package fabrication [4-5]. In this work, a dedicated simulation system to MCM interconnection networks has been developed.

Parameter Calculation
Traditionally, interconnections are simply treated as short-circuit or lumped circuit components. However, as circuit speed and density increase, interconnections become electrically long and rise times of the waveforms are comparable to the time of flight across the line. In this case, the wires must be modeled as transmission lines. Moreover, significant reflections can be generated from the discontinuities of lines, such as step in width, right-angle bend, Tjunction, ..., etc. To analyze the effects of these discontinuities [6], equivalent circuit models must be established.

In order to analyze the crosstalk among interconnections, wires can be modeled as multiconductor transmission lines. The configuration of a multiconductor transmission line is shown in Figure 1. The time-domain transmission-line equations are given by

$$
\frac{\partial \hat{v}(x, t)}{\partial x}=-[R] \mathbf{i}(x, t)-[L] \frac{\partial(x, t)}{\partial x}
$$

\footnotetext{
* This work was supported by the National Science Council of Republic of China under Contract NSC86-2221E002-065.
} 
$\frac{\partial(x, t)}{\partial x}=-[G] \mathbf{v}(x, t)-[C] \frac{\partial \mathbf{v}(x, t)}{\partial x}$,

where

$\mathbf{v}(x, t)=\left[v_{1}(x, t), v_{2}(x, t), \ldots, v_{N}(x, t)\right]^{T} . \quad 0 \leq X \leq D$

and

$\mathbf{i}(x, t)=\left[i_{1}(x, t), i_{2}(x, t), \ldots, i_{N}(x, t)\right]^{\mathrm{T}} \quad 0 \leq x \leq D$

are the vector of line voltages and line currents;

respectively,

$[R]$ is resistance matrix per unit length, in $\Omega / m$,

$[L]$ is inductance matrix per unit length, in $\mathrm{H} / \mathrm{m}$,

[G] is conductance matrix per unit length, in $S / \mathrm{m}$, and

$[C]$ is capacitance matrix per unit length, in $F / m$.

The frequency-domain transmission-line equations of a multiconductor transmission line are given below

$\frac{d \mathbf{V}(x)}{d x}=-[R] \mathbf{I}(x)-j \omega[L] \mathbf{I}(x)$
$\frac{d \mathbf{I}(x)}{d x}=-[G] \mathbf{V}(x)-j \omega[C] \mathbf{V}(x)$

where $\mathbf{V}(\mathrm{x})$ is the vector of complex line voltages and $I(x)$ is the vector of complex line currents.

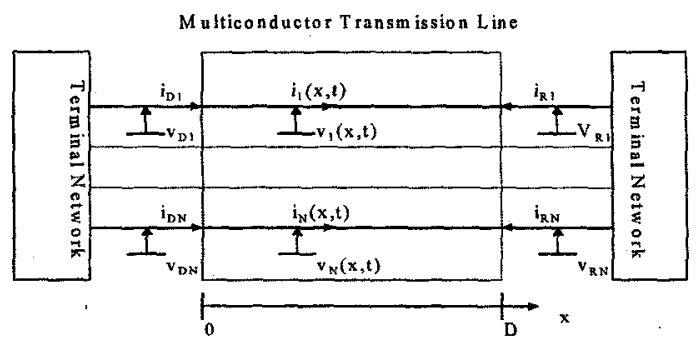

Figure 1. A multiconductor transmission line with terminal networks

From equation (3) and (4), we can derive the wave equations of a multiconductor transmission line to be

$$
\begin{aligned}
& \frac{d^{2} \mathbf{V}(x)}{d x^{2}}=-[Z][Y] \mathbf{V}(x) \\
& \frac{d^{2} \mathbf{I}(x)}{d x^{2}}=-[Y][Z] \mathbf{I}(x)
\end{aligned}
$$

where

$$
\begin{aligned}
& {[Z]=[R]+j \omega[L]} \\
& \text { and } \\
& {[Y]=[G]+j \omega[C]}
\end{aligned}
$$

are per-unit-length impedance matrix and per-unitlength admittance matrix, respectively.
Before solving the transmission equations above, we must first determine the matrix parameter $[R],[L],[G]$, and $[C]$. Therefore, a numerical method for evaluating the capacitance and inductance matrix is investigated. Once these matrix parameters are determined, we can evaluate the time-domain response of multiconductor transmission lines by modified nodal analysis along with numerical inversion of Laplace transform.

A computer program, i.e., parameter calculator, based on the boundary element method has been written to compute the per-unit-length capacitance and inductance matrices of coupled microstrip lines. In this work, we will briefly illustrate the algorithm of this parameter calculator under the moment of method and compare the computation results with that obtained from the closed-form expressions presented in [7].

\section{Simulation of Interconnection Networks}

Consider the wave equations $\{5\}$ and (6) for a multi-conductor transmission line. Let $\gamma_{1}^{2}, \gamma_{2}^{2}, \ldots$, $\gamma_{\mathrm{N}}^{2}$ denote the eigenvalues of the $\mathrm{N}$ by $\mathrm{N}$ matrix $[\mathrm{Z}][\mathrm{Y}]$, and $\mathrm{v}_{1}, \mathrm{v}_{2}, \ldots, \mathbf{v}_{\mathrm{N}}$ be the corresponding eigenvectors. Then, we can derive the following matrices, namely,

$\left[S_{v}\right]=\left[v_{1}, v_{2}, \ldots, v_{N}\right]$

$[\Gamma]=$ diagonal $\left\{\gamma_{1}, \gamma_{2}, \ldots, \gamma_{N}\right\}$

$\left[S_{j}\right]=[Z]^{-1}\left[S_{\nu}\right][\Gamma]$

$[E(x)]=$ diagonal $\left\{\exp \left(-\gamma_{1} x\right), \exp \left(-\gamma_{2} x\right), \ldots, \exp (-\right.$ $\left.\left.\gamma_{N} x\right)\right\}$

The solutions to wave equations are given by [8], and are written as follows

$\boldsymbol{V}(x)=\left[S_{v}\right][E(x)] \boldsymbol{C}_{1}+\left[S_{v}\right][E(x)]^{-1} \boldsymbol{C}_{2}$

$I(x)=\left[S_{i}\right][E(x)] C_{1}+\left[S_{i}\right][E(x)]^{-1} C_{2}$,

where $C_{1}$ and $C_{2}$ are constant vectors. At the terminals $x_{i}=0$ and $x_{f}=D, V(x)$ and $I(x)$ are related by

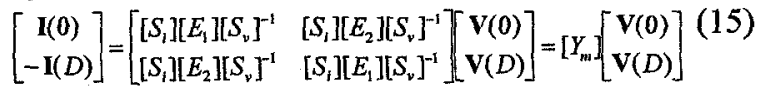
where

$$
\left[E_{1}\right]=\text { diagonal }\left\{\frac{e^{\gamma_{m} D}+e^{-\gamma_{m} D}}{e^{\gamma_{m} D}-e^{-\gamma_{m} D}}, m=1,2, \cdots, N\right\}
$$




$$
\left[E_{2}\right]=\operatorname{diagonal}\left(\frac{-2}{e^{\gamma_{m} D}-e^{-\gamma_{m} D}} \quad m=1,2, \ldots, N\right\}
$$

The matrix $\left[\mathrm{Y}_{\mathrm{m}}\right]$ is just the MNA (modified nodal admittance) matrix of a multiconductor transmission line.

A simulation program based on modified nodal formulation and numerical inversion of Laplace transform has been written to evaluate the timedomain response of $\mathrm{MCM}$ interconnections. This simulator supports six kinds of circuit components, including resistors, capacitors, inductors, independent voltage sources, two-conductor transmission lines, and multiconductor transmission lines. The inputs to the simulator are a circuit file describing the network to be analyzed. The output of the simulator is a file including all nodal voltage waveforms.

\section{Algorithm of the Circuit Simulator} follows.

The algorithm of the circuit simulator is as

Step 1: Process the input file and store the parameters of each circuit component.

Step2: For each two-conductor transmission line, compute its per-unit-length capacitance, inductance, and resistance.

Step3: For each multi-conductor transmission line, compute its per-unit-length capacitance and inductance matrices.

Step 4: Compute the time-domain response $v_{\pi}(t)$ by numerical inversion of Laplace transform with $M$ $=10, \mathrm{~N}=8$ :

$$
\begin{aligned}
& \quad A C C=0 ; \\
& \text { for }(i=1 ; \quad i<=5 ; \quad i++) \\
& \left\{\begin{array}{l}
\mathrm{S}=\mathrm{z}_{\mathrm{i}} / \mathrm{t} ;\left(\mathrm{z}_{\mathrm{i}} \text { is the poles }\right)
\end{array}\right.
\end{aligned}
$$

For all linear lumped components, derive the matrices $\left[\mathrm{C}_{\pi}\right]$ and $\left[\mathrm{G}_{\pi}\right]$.

For all independent voltage sources, derive the vector $\mathrm{E}_{\pi}(\mathrm{s})$.

For all two-conductor transmission lines, derive their admittance matrices [ $\left.Y_{t}\right]$

For all multiconductor transmission lines, derive their admittance matrices $\left[\mathrm{Y}_{\mathrm{m}}\right]$ by equation (15).

Compute system matrix $\left[\mathrm{Y}_{\mathrm{s}}(\mathrm{s})\right]$

Compute $\mathrm{V}_{\pi}(\mathrm{s})$

$\mathrm{ACC}=\mathrm{ACC}+\operatorname{Re}\left\{\mathrm{K}_{\mathrm{i}}{ }^{\prime} \mathrm{V}_{\pi}(\mathrm{s})\right\}$;

( $\mathrm{K}_{\mathrm{i}}{ }^{\prime}$ is the residues)

\}

$$
\mathrm{v}_{\pi}(t)=(-1 / \mathrm{t}) * \mathrm{ACC}
$$

End

\section{Example}

Consider the circuit as shown in Figure 2(a). This circuit contains three coupled microstrip lines, an isolated microstrip line, and an isolated stripline. The parameters of microstrip line and stripline are listed in Table 1 and the-waveform of voltage source is shown in Figure 2(b). Note that this circuit contains all of the components supported by our simulator and serves as a good example to demonstrate our simulator.

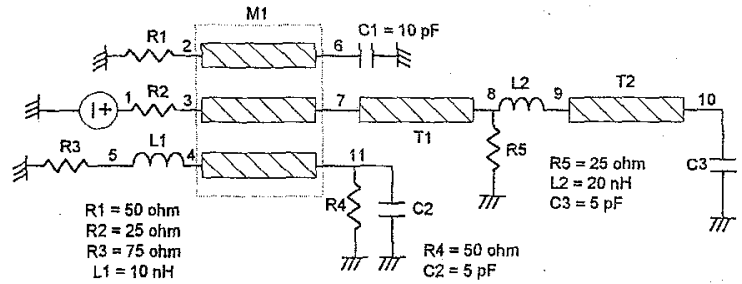

(A)

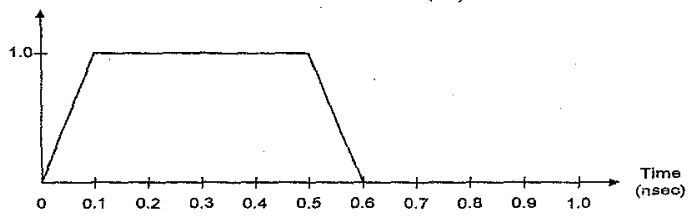

(B)

Figure 2. (a) A circuit containing coupled microstrip lines, isolated microstrip line, and stripline, and (b) waveform of voltage source.

The voltage waveforms at nodes 6,10 , and 11 are shown in Figure 3. Note that there are small differences between the simulation results of HSPICE and those of our simulator. This is due to the difference between the electrical models of interconnections used by simulators. Wires are modeled as cascaded lumped circuits in HSPICE while as transmission lines in our simulator. As shown in Figure 3, the simulation results match acceptably. Total execution time of this circuit is given below:

Our simulator on Pentium $133^{\circledR}: 7.25$ seconds HSPICE on SPARC 20 ${ }^{\circledR} \quad 7.02$ seconds.

Table 1. Geometric and constitutive parameters of microstrip lines and stripline in Figure 2.

\begin{tabular}{|l|l|l|l|}
\hline & $\mathrm{M} 1$ & $\mathrm{~T} 1$ & $\mathrm{~T} 2$ \\
\hline Conductor Width & $5 \mu \mathrm{m}$ & $10 \mu \mathrm{m}$ & $8 \mu \mathrm{m}$ \\
\hline Conductor Thickness & $2 \mu \mathrm{m}$ & $2 \mu \mathrm{m}$ & $2 \mu \mathrm{m}$ \\
\hline Spacing between Conductors & $10 \mu \mathrm{m}$ & & \\
\hline Conductor Length & $5 \mathrm{~mm}$ & $4 \mathrm{~mm}$ & $10 \mathrm{~mm}$ \\
\hline Dielectric Thickness & $5 \mu \mathrm{m}$ & $5 \mu \mathrm{m}$ & $5 \mu \mathrm{m}$ \\
\hline Relative Dielectric Constant & 3.5 & 3.5 & 3.5 \\
\hline
\end{tabular}




\begin{tabular}{|l|l|l|l|}
\hline $\begin{array}{l}\text { Spacing between Reference } \\
\text { Planes }\end{array}$ & & & $12 \mu \mathrm{m}$ \\
\hline
\end{tabular}

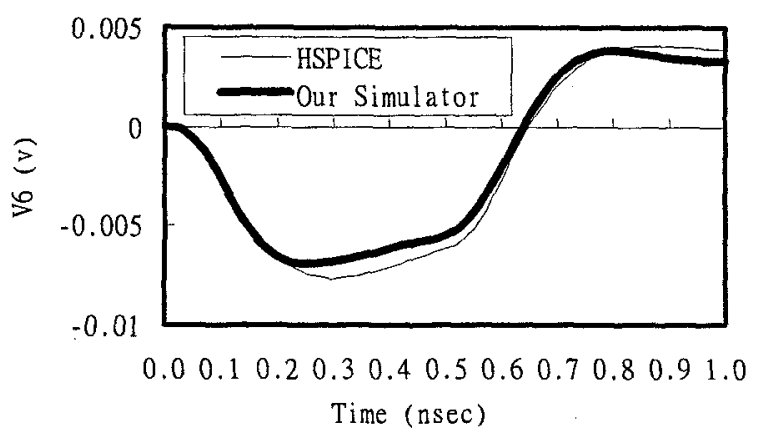

(A)

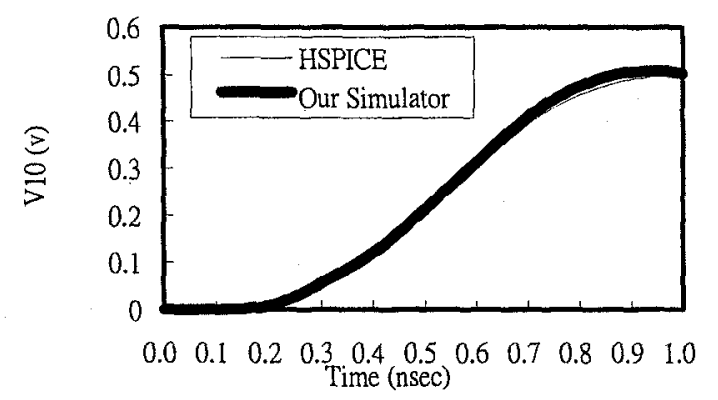

(B)

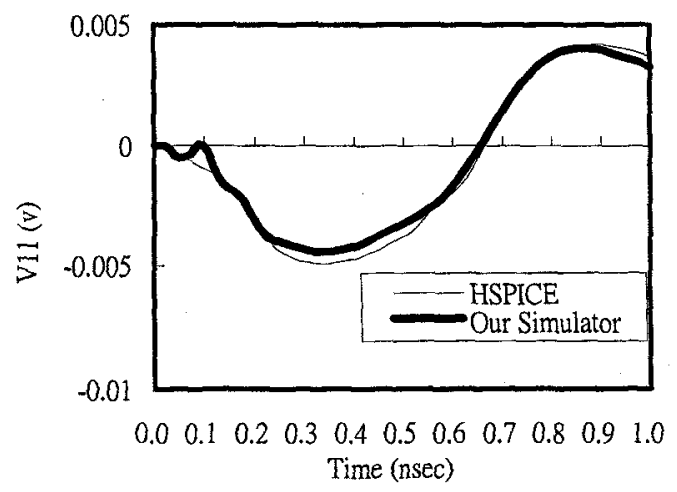

(C)

Figure 3. Simulation results of the circuit in Figure 2, (a) V6, (b) V10, and (c) V11.

\section{Optimization of MCM Interconnections}

To reduce the transmission-line effects such as signal propagation delay, reflection, and crosstalk, we must find the optimal geometric parameters of interconnections. Since it is extremely difficult and time-consuming to find these optimal values by tryand-error, a circuit optimizer is required to optimize the interconnection design automatically. We will present the basic definitions and concepts of optimization problems. Also, one-dimensional and multidimensional minimization algorithms for the optimization problems are described. Based on the minimization algorithms, we have developed a circuit optimizer. The experimental results with this optimizer are presented.

Once a design problem is formulated as a minimax problem, various minimization methods can be applied to find the optimal solutions. In this section, we will present the algorithm of these minimization methods. Since the minimization techniques as presented in this section can solve only unconstrained problems, we must convert all constrained parameters in the design to unconstrained ones before applying these methods.

Almost all modern minimization techniques are based on determining a sequence of vector $x_{k}$ such that

$U\left(x_{0}\right)>U\left(x_{1}\right)>\ldots>U\left(x_{k}\right)$

This sequence will converge to the minimum $\mathbf{x}^{*}$ if the function is convex. At any point $x_{k}$, we must choose a direction $s_{k}$ and take a step of length $d_{k}$ along this direction to reach a new point $x_{k+1}$, that is, $x_{k+1}=x_{k}+d_{k} s_{k}$

The main difference between various optimization methods is in the algorithm of determining $d_{k}$ and $s_{k}$.

A general algorithm for the minimization methods based on equation (18) is described as follows.

$\mathrm{U}(\mathbf{x})$ : An objective function to be minimized.

Step 1:Set $k=0$ and select a starting point $x_{0}$.

Step 2: Compute $U\left(x_{k}\right)$ and $\nabla U\left(x_{k}\right)$ (if the method requires the gradient).

Step 3: Determine the search direction $\mathbf{s}_{\mathrm{k}}$.

Step 4: Normalize the search vectors $s_{k}$ to unit length.

Step 5: In the direction $s_{k}$, find a step length $d_{k}$ such that either

$$
\mathrm{U}\left(\mathbf{x}_{\mathrm{k}}+\mathrm{d}_{\mathrm{k}} \mathbf{s}_{\mathrm{k}}\right)<\mathrm{U}\left(\mathbf{x}_{\mathrm{k}}\right)
$$

or

$\mathrm{U}\left(\mathrm{x}_{\mathrm{k}}+\mathrm{d}_{\mathrm{k}} \mathbf{s}_{\mathrm{k}}\right)$ is the minimum in the direction $\mathbf{s}_{\mathrm{k}}$.

Step 6: Obtain

$$
\begin{aligned}
& \Delta \mathbf{x}_{\mathrm{k}}=\mathrm{d}_{\mathrm{k}} \mathbf{s}_{\mathrm{k}} \\
& \mathbf{x}_{\mathrm{k}+1}=\mathbf{x}_{\mathrm{k}}+\Delta \mathbf{x}_{\mathrm{k}}
\end{aligned}
$$

Step 7: If $\left|U\left(x_{k+1}\right)-U\left(x_{k}\right)\right|<\varepsilon_{1}$ and $\left\|\Delta x_{k}\right\|<\varepsilon_{2}$, stop. Otherwise, set $k=k+1$ and go to step 2 . 
As mentioned in the multidimensional minimization, we require an algorithm to determine the search direction $\mathbf{s}_{\mathrm{k}}$. The choice of search directions is usually the most difficult part in the theory of minimization algorithms, and many considerations must be taken into account. Depending on whether the gradient of the objective function is used to determine the search directions, multidimensional minimization method can be classified into two groups, such as direct search methods and gradient methods. In this section, we will present a direct search method proposed by Powell [9]. The modified algorithm of Powell's method is given below.

$U(x)$ : An objective function of $N$ variables to be minimized.

Step 1: Choose an initial point $\mathbf{P}_{0}$ and a set of directions $\mathbf{u}_{\mathrm{i}}$ initialized to the basis vectors $\mathbf{e}_{\mathrm{i}}$, for $\mathrm{i}=1,2, \ldots, \mathrm{N}$.

Step 2: For $1,2, \ldots, N$, move from $\mathbf{P}_{\mathrm{i}-1}$ to the

minimum along direction $\mathbf{u}_{i}$ and call this point $\mathbf{P}_{i}$.

If the magnitude of decrement of the objective function along $\mathbf{u}_{i}$ is the largest, record index $i$ and store the magnitude of decrement in $\Delta$.

Step 3: If $\left|\mathrm{U}\left(\mathbf{P}_{0}\right)-\mathrm{U}\left(\mathbf{P}_{\mathrm{N}}\right)\right|<\varepsilon_{1}$ and $\left\|\mathbf{P}_{0}-\mathbf{P}_{\mathrm{N}}\right\|<\varepsilon_{2}$, stop.

Step 4: Set

$$
\begin{aligned}
& \mathrm{U}_{1}=\mathrm{U}\left(\mathbf{P}_{0}\right) \\
& \mathrm{U}_{2}=\mathrm{U}\left(\mathbf{P}_{\mathrm{N}}\right) \\
& \mathrm{U}_{3}=\mathrm{U}\left(2 \mathbf{P}_{\mathrm{N}}-\mathbf{P}_{0}\right)
\end{aligned}
$$

Step 5: Test whether

$$
\left(U_{3} \geq U_{1}\right)
$$

Or

$$
2\left(U_{1}-2 U_{2}+U_{3}\right)\left(U_{1}-U_{2}-\Delta\right)^{2} \geq \Delta\left(U_{1}-U_{3}\right)^{2} \text {. }
$$

If either of these conditions holds, retain the old set of directions $\left\{\mathbf{u}_{1}, \mathbf{u}_{2}, \ldots, \mathbf{u}_{\mathrm{N}}\right\}$ and use $\mathbf{P}_{\mathrm{N}}$ as the new $\mathbf{P}_{0}$.

Go to step 2.

Step 6: If neither of the tests in step 4 holds, set

$$
\mathbf{v}=\mathbf{P}_{\mathrm{N}}-\mathbf{P}_{0}
$$

and move from $\mathbf{P}_{\mathrm{N}}$ to the minimum along the direction $\mathbf{v}$. Take this minimum point as the new $\mathbf{P}_{0}$ and add $\mathbf{v}$ to the set of directions $\left\{\mathbf{u}_{1}, \mathbf{u}_{2}, \ldots, \mathbf{u}_{\mathrm{N}}\right\}$, dropping the direction in which the largest increment was made. Go to step 2 .

Note that step 2 of the Algorithm, onedimensional search is carried out in each direction $\mathbf{u}_{i}$. We can use the quadratic interpolation method to find the minimum along each direction.

\section{Experimental Results}

Based on the quadratic interpolation method and Powell's method, we have developed a circuit optimizer. This optimizer can help us find the optimal geometric parameters of interconnections. In order to illustrate the optimizer; let's consider the circuit as shown in Figure 4. We want to minimize the crosstalk at node 5 and propagation delay at node 6 . The ranges of conductor width $\mathrm{w}$ and conductor length $\mathrm{D}$ are shown below.

$$
\begin{aligned}
& 1 \mu m \leq w \leq 5 \mu m \\
& 5 m m \leq D \leq 2 \mathrm{~cm}
\end{aligned}
$$

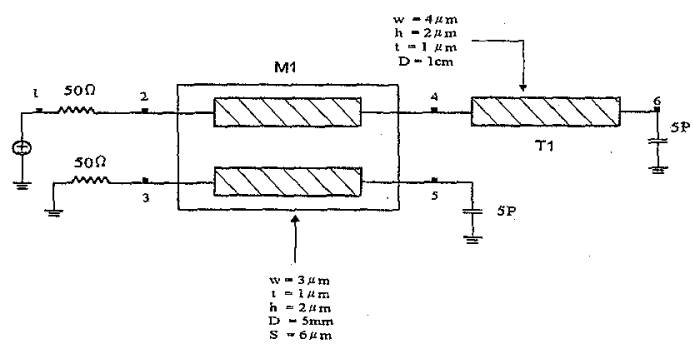

Figure 4. An example circuit for optimization.

The voltage waveforms of node 5 and node 6 are shown in Figure 5. After optimization, the crosstalk and propagation delays are indeed reduced and the resulting geometric parameters of interconnections are shown in Table 2.

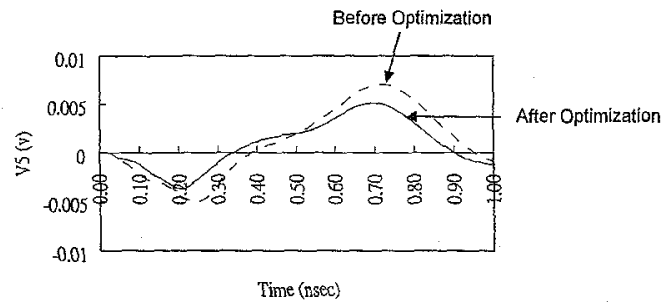

(A)

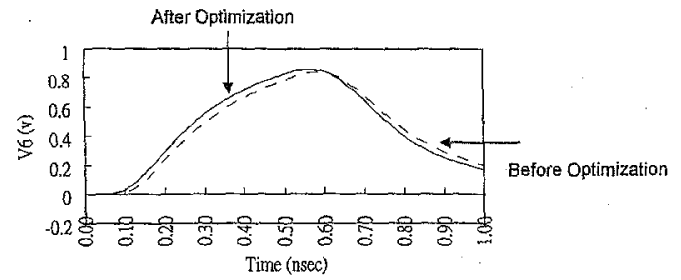

(B)

Figure 5. Voltage waveforms of (a) node 5 and (b) node 6. 
Table 2 Geometric parameters after optimization.

\begin{tabular}{|l|l|l|}
\hline & $\mathrm{M} 1$ & $\mathrm{~T} 1$ \\
\hline Width & $5 \mu \mathrm{m}$ & $1 \mu \mathrm{m}$ \\
\hline Length & $5 \mathrm{~mm}$ & $5 \mathrm{~mm}$ \\
\hline
\end{tabular}

\section{Conclusions}

In this work, we develop a dedicated simulation system for MCM interconnection networks. This simulation system consists of three parts; namely, parameter calculator, circuit simulator, and circuit optimizer. The parameter calculator computes the transmission-line parameters of microstrip lines and striplines. Computational results have been compared with those of empirical formulas and references. The relative errors are smaller than $6 \%$. Based on the parameter calculator, a circuit simulator for MCM interconnection networks has been built. This simulator supports six circuit components, including resistors, capacitors, inductors, independent voltage sources with trapezoidal waveforms, two-conductor transmission lines, and multiconductor transmission lines. The major advantage of our simulator over HSPICE is that our simulator can simulate more than five coupled transmission lines. However, the speed of our simulator on PC Pentium ${ }^{\circledR} 133$ is a little slower than HSPICE on SUN SPARC ${ }^{\circledR} 20$. In order to help the package designer to find the optimal parameters of interconnections in the design of MCM interconnections, a circuit optimizer has been developed. Good agreement was obtained.

\section{References}

[1] R. W. Johnson, R. K. F. Teng, and J. W. Balde, Multichip Modules: System Advantages, Major Constructions, and Materials Technologies, New York: IEEE Press, 1991.

[2] Y. Yang, and J. R. Brews, "Guidelines for High- performance Electronic Package Interconnections - A Simple Approach," IEEE Trans. Components, Hybrids, Manuf. Technical. - Part B, vol. CHMT-19, pp. 230-237, Feb. 1996.

[3] Y. Yang, and J. R. Brews, "Guidelines for Highperformance Electronic Package Interconnections Approach for Strong Coupling," IEEE Trans. Components, Hybrids, Manuf. Technical. - Part B, vol. CHMT-19, pp. 372-381, May 1996.

[4] T. Simunic, J. W. Rozenblit, and J. R. Brews, "VLSI Interconnect Design Automation Using Quantitative and Symbolic Techniques," IEEE Trans. Components, Hybrids, Manuf. Technical. - Part B, vol. CHMT-19, pp. 803-812, Nov. 1996.

[5] J. R. Griffith, E. Chiprout, Q. J. Zhang, and M. S. Nakhla, "A CAD Framework for Simulation and Optimization of High-speed VLSI Interconnections," IEEE Trans. Circuits Syst, vol. CAS-39, pp. 893-906, Nov. 1992.

[6] K. C. Gupta, R. Garg, and I. Bahl, and P. Bhartia, "Microstrip Lines and Slot lines", Computer-Aided Design of Microwave Circuits, Chapter 3 and 4, Artech House Inc. Boston, 1996.

[7] C. Wei, R. F. Harrington, J. R. Mautz, and T. K. Sarkar, "Multiconductor Transmission Lines in Multilayered Dielectric Media," IEEE Trans. Microwave Theory Tech., vol. MTT-32, pp. 439-450, Apr. 1984.

[8] J. R. Griffith, and M. S. Nakhla, "Time-Domain Analysis of Lossy Coupled Transmission Lines," IEEE Trans. Microwave Theory Tech., vol. MTT-38, pp. 1480-1487, Oct. 1990.

[9] M. J. D. Powell, "An Efficient Method for Finding the Minimum of a Function of Several Variables without Calculating Derivatives," Computer J., vol. 7, pp. 149-154, July 1964. 